\title{
Pathologic von Willebrand factor degradation with a left ventricular assist device occurs via two distinct mechanisms: Mechanical demolition and enzymatic cleavage
}

\author{
Carlo R. Bartoli, MD, PhD, David J. Restle, BSE, David M. Zhang, Michael A. Acker, MD, and \\ Pavan Atluri, MD
}

\begin{abstract}
Objectives: Bleeding is an important source of morbidity in patients with a left ventricular assist device. Evidence suggests a major role for von Willebrand factor. However, limited data exist to explain the mechanism(s) of von Willebrand factor degradation during left ventricular assist device support. We investigated whether left ventricular assist device-related shear stress and a disintegrin and metalloproteinase with a thrombospondin type 1 motif, member 13 (ADAMTS-13, the von Willebrand factor protease) altered von Willebrand factor metabolism.
\end{abstract}

\begin{abstract}
Methods: Whole blood was collected from patients $(\mathrm{n}=8)$ with a left ventricular assist device. von Willebrand factor multimers and degradation fragments were characterized with electrophoresis and immunoblotting. To investigate mechanisms, an in vitro model was developed to generate the supraphysiologic shear stress of a continuousflow left ventricular assist device. Normal human blood $(\mathrm{n}=8)$ was cycled in a laboratory vortexer $(\sim 2400 \mathrm{rpm}$, shear stress $\sim 175$ dyne $/ \mathrm{cm}^{2}, 4$ hours) to reproduce the pathologic degradation of von Willebrand factor that occurs during left ventricular assist device support. To investigate the specific mechanistic roles of shear stress and ADAMTS-13 in von Willebrand factor degradation, purified von Willebrand factor protein \pm ADAMTS-13 protease were exposed to supraphysiologic shear stress in the vortexer. von Willebrand factor multimers and 11 von Willebrand factor degradation fragments were characterized with electrophoresis and immunoblotting.
\end{abstract}

Results: Left ventricular assist device support reduced large von Willebrand factor multimers and significantly increased 10/11 von Willebrand factor degradation fragments $(P<.05)$. Normal human blood exposed to supraphysiologic shear stress in the vortexer demonstrated the same profile of von Willebrand factor degradation fragments as in a patient with a left ventricular assist device. Supraphysiologic shear stress alone caused modest mechanical demolition of large von Willebrand factor multimers into smaller multimers but did not greatly generate von Willebrand factor fragments. In the presence of supraphysiologic shear stress, ADAMTS-13 completely eliminated large von Willebrand factor multimers and generated statistically significant amounts of $11 / 11$ von Willebrand factor degradation fragments $(P<.05)$. The profile of von Willebrand factor fragments generated was identical to the profile that was observed in vivo in patients with a left ventricular assist device.

Conclusions: Supraphysiologic shear stress alone causes physical demolition of large von Willebrand factor multimers into smaller von Willebrand factor multimers. In the setting of supraphysiologic shear stress, ADAMTS-13 cleaves large von Willebrand factor multimers into von Willebrand factor degradation fragments. ADAMTS-13 may be a therapeutic target to reduce von Willebrand factor degradation and bleeding complications in patients with a left ventricular assist device. (J Thorac Cardiovasc Surg 2015;149:281-9)

See related commentary on pages $289-90$.

From the Division of Cardiovascular Surgery, Hospital of the University of Pennsylvania, Philadelphia, Pa.

Disclosures: Michael A. Acker reports consulting fees from Thoratec Inc and Heartware Inc. All other authors have nothing to disclose with regard to commercial support.

Received for publication July 20, 2014; revisions received Aug 21, 2014; accepted for publication Sept 4, 2014; available ahead of print Nov 12, 2014.

Address for reprints: Carlo R. Bartoli, MD, PhD, Division of Cardiovascular Surgery, Hospital of the University of Pennsylvania, Stemmler Hall, Room 351, Philadelphia, PA 19104 (E-mail: carlo.bartoli@uphs.upenn.edu).

$0022-5223 / \$ 36.00$

Copyright $\odot 2015$ by The American Association for Thoracic Surgery

http://dx.doi.org/10.1016/j.jtcvs.2014.09.031
Over the past decade, left ventricular assist devices (LVADs) have emerged as a standard, long-term therapy for adult patients with advanced heart failure. With more than 6000 patients studied, the Interagency Registry for Mechanically Assisted Circulatory Support documented a recent 2-year survival of $70 \%{ }^{1}$ However, hematologic pathologies that are related to abnormal blood flow from the LVAD may limit longterm survival.

For example, numerous reports have documented abnormal von Willebrand factor ( $\mathrm{vWF}$ ) metabolism and frequent nonsurgical bleeding during LVAD support. ${ }^{2}$ In these studies, an acquired von Willebrand syndrome developed in patients with an LVAD. ${ }^{3-10}$ As a result, impaired 


\section{Abbreviations and Acronyms \\ ADAMTS-13 $=$ a disintegrin and metalloproteinase with a thrombospondin type 1 motif, member 13

$\begin{array}{ll}\text { LVAD } & =\text { left ventricular assist device } \\ \text { SDS } & =\text { sodium dodecyl sulfate } \\ \text { TBS } & =\text { Tris-buffered saline } \\ \text { vWF } & =\text { von Willebrand factor }\end{array}$

primary hemostasis through the vWF-platelet pathway ${ }^{11}$ produced a bleeding diathesis in $30 \%$ to $75 \%$ of patients with an LVAD. ${ }^{4-6,9,10}$

The mechanisms of pathologic vWF degradation with an LVAD are unclear. Preliminary experimental data have suggested that nonphysiologic flow characteristics of LVADs and enzymatic degradation by the vWF protease, a disintegrin and metalloproteinase with a thrombospondin type 1 motif, member 13 (ADAMTS13), alter vWF metabolism. ${ }^{11,12}$ However, clinical observations of normal plasma ADAMTS-13 values in LVAD patients have suggested that ADAMTS-13 does not play a major role in LVAD-related bleeding. 5,6,10

The roles of shear stress and ADAMTS-13 in LVADrelated bleeding have not been defined. Particularly, the role of ADAMTS-13 is unclear. This may be of great clinical importance because ADAMTS-13 may be pharmacologically inhibited. The goal of this study was to investigate the mechanistic roles of shear stress and ADAMTS-13 in LVAD-associated degradation of vWF.

\section{MATERIALS AND METHODS}

This study was conducted with approval from the University of Pennsylvania blood bank and Institutional Review Board (\#818944, "Novel Model of LVAD-Like von Willebrand Factor Degradation for the Investigation of Bleeding in Patients With an LVAD”). All patients provided informed consent.

\section{Project Overview and Experimental Design}

Whole blood was collected from patients at baseline and after the implantation of a continuous-flow LVAD $(n=8)$. In these patients, the profile of vWF multimers and degradation fragments was characterized with electrophoresis and immunoblotting.

To investigate the mechanisms of vWF degradation, an in vitro model was developed to reproduce the supraphysiologic shear stress of a continuous-flow LVAD. Volunteer donor human blood $(\mathrm{n}=8)$ was cycled in a laboratory vortexer to produce the pathologic degradation of vWF that occurs with an LVAD. To investigate the mechanistic roles of shear stress and ADAMTS-13, purified vWF protein alone or in the presence of recombinant ADAMTS-13 protein was exposed to supraphysiologic shear stress in the vortexer $(n=5)$. vWF multimers and vWF degradation fragments were characterized with electrophoresis and immunoblotting and compared with the profile observed in patients with an LVAD.

\section{Blood Sample Collection}

Paired blood samples were obtained from patients with an $\operatorname{LVAD}(n=8)$ at baseline, before the implantation of a continuous-flow LVAD (HeartMate
II, Thoratec Corp, Pleasanton, Calif [ $=4$ ]; HeartWare HVAD, HeartWare Inc, Framingham, Mass $[n=4]$ ), and 7 days after LVAD implantation. In parallel, blood samples were obtained from volunteer donors via venipuncture $(\mathrm{n}=8)$. Fresh whole blood $(\sim 15 \mathrm{~mL})$ was anticoagulated in $6 \mathrm{~mL}$ buffered sodium heparin blood tubes (BD Biosciences, Franklin Lakes, $\mathrm{NJ}$ ) and used for in vitro experiments. Plasma was frozen and stored at $-80^{\circ} \mathrm{C}$ for electrophoresis and immunoblotting.

\section{In Vitro Model of Supraphysiologic Shear Stress to Induce Left Ventricular Assist Device-Associated von Willebrand Factor Degradation}

To investigate protein-specific mechanisms of vWF degradation, an in vitro model that reproduced the pathologic degradation of vWF with an LVAD was validated. ${ }^{13}$ Volunteer donor human blood samples $(\mathrm{n}=8$, $1 \mathrm{~mL}$ ) were placed in $2 \mathrm{~mL}$ cryogenic tubes (Thermo Fisher Scientific, Inc, Rockford, Ill). Samples were exposed to a continuous cyclone spin for 4 hours at $2400 \mathrm{rpm}$ in a laboratory vortexer (Vortex Genie-2; Scientific Industries, Bohemia, NY) at room temperature. Paired baseline samples were stored at room temperature for 4 hours. Samples were frozen and stored at $-80^{\circ} \mathrm{C}$ for electrophoresis and immunoblotting.

The vortexer model was developed to generate supraphysiologic shear stress like an LVAD. With rotational speeds of 600 to $3200 \mathrm{rpm}$, the vortexer imparts an in-plane orbital motion on the cylindrical tube containing blood samples. As a result, blood in the tube undergoes rigid body rotation around the center of the well. ${ }^{14}$ The magnitude of shear stress on the bottom surface of the well may be estimated with a 2-dimensional extension of Stokes' Second Problem. ${ }^{14,15}$ With this approximation in which $\mathrm{R}_{\mathrm{g}}$ is the radius of gyration, $\rho$ is the density, $\omega$ is the angular velocity, and $\mu$ is the viscosity of the fluid, the magnitude of shear stress $(\tau)$ is equal to the following:

$$
\tau=R_{g} \omega^{3 / 2} \sqrt{\rho \mu}
$$

Similar to LVADs, which are reported to generate shear stress 1 to 2 orders of magnitude greater than physiologic values, ${ }^{16}$ the vortexer produced shear stress of approximately $175 \mathrm{dyne} / \mathrm{cm}^{2}, 4$ to 12 times greater than the normal physiologic range of 15 to $40 \mathrm{dyne} / \mathrm{cm}^{2} .{ }^{17}$

\section{Roles of Shear Stress and ADAMTS-13 in Left Ventricular Assist Device-Associated von Willebrand Factor Degradation}

Purified native human vWF (Thermo Fisher Scientific, Inc) and recombinant full-length human ADAMTS-13 (R\&D Systems, Minneapolis, Minn) were obtained. Purified vWF alone $(10 \mu \mathrm{g} / \mathrm{mL})^{18}$ and purified vWF $(10 \mu \mathrm{g} / \mathrm{mL})$ plus purified ADAMTS-13 $(0.5 \mu \mathrm{g} / \mathrm{mL})^{19}$ were prepared in a buffered physiologic solution (EGM-2; Lonza, Basel, Switzerland). Samples were exposed to continuous supraphysiologic shear stress $\left(\sim 175 \mathrm{dyne} / \mathrm{cm}^{2}\right)$ for 4 hours in the vortexer as described previously.

\section{Agarose Gel Electrophoresis and Immunoblotting for High-Molecular-Weight von Willebrand Factor Fragments}

The relative size of high-molecular-weight plasma vWF multimers was analyzed by standard immunoblotting techniques as previously described. $^{11,12}$ Plasma samples were each combined 1:20 with loading buffer ( $1 \mathrm{mmol} / \mathrm{L}$ ethylenediaminetetraacetic acid, $10 \mathrm{mmol} / \mathrm{L}$ Tris-HCL, $8 \mathrm{~mol} / \mathrm{L}$ urea, $2 \%$ sodium dodecyl sulfate [SDS], bromophenol blue, $\mathrm{pH}$ 8.0). Samples were heated in a $60^{\circ} \mathrm{C}$ water bath for 20 minutes. Samples were loaded into $1.8 \%$ agarose-SDS gels $(8.3 \times 8 \mathrm{~cm} \times 1.5 \mathrm{~mm}, 1 \%$ SDS, $0.375 \mathrm{~mol} / \mathrm{L}$ Tris HCl; Life Technologies, Grand Island, NY). Electrophoresis was performed at $60 \mathrm{~V}$ for 1.75 hours at $4{ }^{\circ} \mathrm{C}$ in $1 \times$ TrisAcetate SDS running buffer (Life Technologies) in an XCell SureLock Mini-Cell Electrophoresis System (Life Technologies). 
Proteins were transferred for 8.5 minutes with the iBlot dry transfer device onto iBlot PVDF Gel Transfer Stacks (Life Technologies). Blots were blocked for 1 hour in milk blocking buffer $(5 \%$ dried milk powder in Tris-buffered saline [TBS], 0.05\% Tween-20; Thermo Fisher Scientific, Inc). Blots were incubated with a rabbit anti-human vWF primary antibody (1/500; Dako, Carpinteria, Calif) overnight at $4{ }^{\circ} \mathrm{C}$ in TBS-Tween $/ 5 \%$ milk. Blots were washed in TBS-Tween and incubated with goat anti-rabbit immunoglobulin-G horseradish peroxidase-conjugated secondary antibody (1/3000; Cell Signaling, Danvers, Mass) for 2 hours at room temperature in TBS-Tween $/ 5 \%$ milk. Blots were developed with Luminata Forte Western Blot HRP Substrate (Millipore, Billerica, Mass) and imaged with an ImageQuant LAS 4000 (GE Healthcare, Piscataway, NJ).

\section{Polyacrylamide Gel Electrophoresis and Immunoblotting for Low-Molecular-Weight von Willebrand Factor Degradation Fragments}

The relative size of low-molecular-weight plasma vWF degradation fragments was analyzed by standard immunoblotting techniques as previously described. ${ }^{11}$ Plasma samples were combined 1:40 in NuPAGE LDS Sample Buffer (Life Technologies). Samples were incubated at $70^{\circ} \mathrm{C}$ for 10 minutes and loaded into NuPAGE 3\%-8\% Tris-Acetate gels. Prestained Precision Plus Protein Kaleidoscope Standard Protein Ladder (Bio-Rad, Hercules, Calif) and HighMark Prestained Standard Protein Ladder (Life Technologies) were loaded. Electrophoresis was performed at $150 \mathrm{~V}$ for 1.25 hours with $1 \times$ Tris-acetate SDS running buffer (Life Technologies). Proteins were transferred, blocked, probed for $\mathrm{vWF}$, and imaged as described earlier.

A protein loading control was performed by immunoblotting for human plasma albumin. Blots were washed and blocked for 1 hour at room temperature in TBS-Tween $/ 5 \%$ milk. Blots were incubated overnight in goat anti-human albumin primary horseradish peroxidase-conjugated antibody (1:5000 in TBS-Tween $/ 5 \%$ milk, $4^{\circ} \mathrm{C}$; Abcam, Cambridge, Mass). Blots were washed and developed with Luminata Classico Western Blot HRP Substrate (Millipore) and imaged with an ImageQuant LAS 4000 (GE Healthcare).

\section{Low-Molecular-Weight von Willebrand Factor Degradation Fragment Immunoblotting Analysis}

Relative levels of vWF degradation fragments were quantified. ImageJ (National Institutes of Health, Bethesda, Md) was used to measure the chemiluminescent intensity of vWF fragment bands that were identified. Percent change was calculated for pre-LVAD versus post-LVAD samples and baseline versus vortexer samples.

\section{ADAMTS-13 Activity During Shear Stress Conditions}

The effect of supraphysiologic shear stress on ADAMTS-13 activity was determined with a commercial FRET kit (American Diagnostica Inc, Stamford, Conn). The assay was performed on samples at baseline and after exposed to continuous supraphysiologic shear stress $\left(\sim 175 \mathrm{dyne} / \mathrm{cm}^{2}\right)$ for 4 hours in the vortexer as described previously. ALEXA fluorochromeconjugated recombinant vWF protein (American Diagnostica) and purified ADAMTS-13 protein $(0.5 \mu \mathrm{g} / \mathrm{mL})$ were added to a buffered physiologic solution (EGM-2). At $37^{\circ} \mathrm{C}$, proteolytic cleavage of the vWF-ALEXA substrate uncoupled ALEXA fluorochromes and resulted in an increase in fluorescence. The increase in fluorescence over time $\left(\mathrm{V}_{\max }\right)$ was measured with a spectrofluorometer. A standard curve was constructed with a known plasma concentration of ADAMTS-13. ADAMTS-13 activity was determined by interpolation of the $\mathrm{V}_{\max }$ values from the standard curve.

\section{Statistics}

GraphPad version 5.00 (Prism; GraphPad Software, Inc, La Jolla, Calif) was used to perform statistical analyses and plot data. Paired Student $t$ tests were used to compare the vWF fragment bands between paired baseline (pre-LVAD or pre-vortexer) and treated (post-LVAD or post-vortexer) plasma samples. Unpaired Student $t$ tests were used to compare the percent change in each vWF degradation fragment between LVAD cases and vortexer samples. All data were presented as mean \pm standard error.

\section{RESULTS}

Profile of von Willebrand Factor Multimers and Degradation Fragments in Patients With a Left Ventricular Assist Device

LVAD support produced a common profile of $\mathrm{vWF}$ multimers and $v W F$ degradation fragments. Patients with an LVAD demonstrated reduced high-molecular-weight vWF multimers and increased low-molecular-weight multimers, as previously observed. ${ }^{4-6,9,10}$ Before LVAD support, 4 low-molecular-weight vWF bands (Figure 1, A, bands $\mathrm{C}, \mathrm{G}, \mathrm{I}, \mathrm{K})$ were clearly observed. After LVAD support, these bands increased significantly $(P<.05)$. Seven additional low-molecular-weight bands (Figure 1, A, bands A, B, D-F, H, J) clearly emerged and increased significantly (Figure $1, B, P<.05$ ).

\section{In Vitro Model of Left Ventricular Assist Device- Like von Willebrand Factor Degradation}

The normal physiologic range of fluid shear stress within the cardiovascular system is 15 to $40 \mathrm{dyne} / \mathrm{cm}^{2} .{ }^{17}$ In comparison, with a blood $\mu$ of $3 \times 10^{-3} \mathrm{~Pa} \cdot \mathrm{s}$, a blood $\rho$ of $1.025 \mathrm{~g} / \mathrm{cm}^{3}$, a $\omega$ of $2400 \mathrm{rpm}$, and an $\mathrm{R}_{\mathrm{g}}$ of $2.5 \mathrm{~mm}$, the vortexer produced a shear stress of approximately 175 dyne $/ \mathrm{cm}^{2}$. In comparison, LVADs are reported to generate shear stress 1 to 2 orders of magnitude greater than physiologic values. ${ }^{16}$

Normal human blood cycled through the vortexer demonstrated the same profile of vWF multimers and degradation fragments as patients with an LVAD (Figure 1, $C$ and $D$ ). The profile of vWF fragments that emerged was nearly identical to the fragments produced in patients with an LVAD, which indicated that this model adequately replicated pathologic vWF metabolism with an LVAD. To confirm that vWF degradation was similar with an LVAD and the vortexer, the percent change for each $v W F$ fragment was compared between patients with an LVAD and vortexer samples. The percent change of 9 of the 11 bands was not statistically different, which suggested that LVADs and the vortexer generated similar levels of $\mathrm{vWF}$ degradation fragments. However, the percent change for 2 bands (Figure 1, bands D and E) was significantly greater $(P<.01)$ in the vortexer samples than in the LVAD patient samples.

\section{Purified von Willebrand Factor Without ADAMTS- 13 During Shear Stress Conditions}

To investigate the mechanisms of altered vWF metabolism, purified $\mathrm{vWF}$ protein and ADAMTS-13 protease 

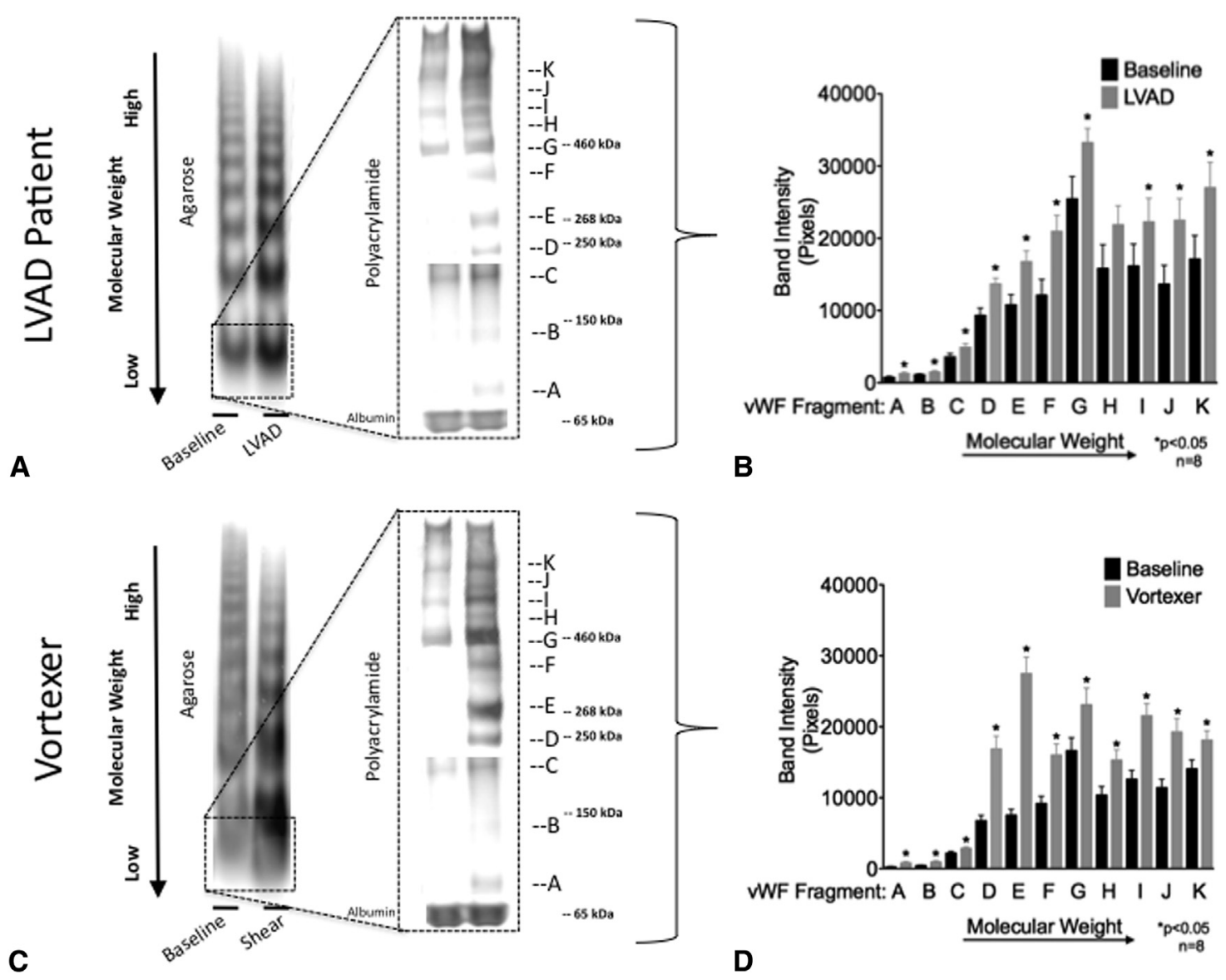

FIGURE 1. A and B, The profile of vWF multimers and degradation fragments was determined in patients undergoing LVAD support. After LVAD support, agarose gel electrophoresis and immunoblotting for vWF revealed a decrease in large vWF multimers and an increase in small multimers compared with baseline (pre-LVAD). Polyacrylamide gel electrophoresis with immunoblotting revealed significantly increased vWF degradation fragments compared with baseline. $\mathrm{C}$ and $\mathrm{D}$, An in vitro model was developed to reproduce the shear stress of a continuous-flow LVAD. Normal human blood exposed to supraphysiologic shear stress demonstrated the same profile of vWF multimers and vWF degradation fragments as patients with an LVAD. LVAD, Left ventricular assist device; $v W F$, von Willebrand factor; $k D a$, kilodalton.

were cycled in the vortexer to simulate the supraphysiologic shear stress of a continuous-flow LVAD (Figure 2). Prior to shear stress, purified $\mathrm{vWF}$ protein demonstrated intact $\mathrm{vWF}$ multimers of all sizes and a relative absence of degradation fragments. Exposure of purified vWF multimers alone (without ADAMTS-13 present) to supraphysiologic shear stress reduced high-molecular-weight vWF multimers and increased low-molecular-weight vWF multimers (Figure 2, $A$ and $B$ ). However, vWF degradation fragments did not demonstrate the same profile or significant increases as observed in patients with an LVAD or when normal human blood (which contains ADAMTS-13) was exposed to supraphysiologic shear stress in the vortexer (Figure 2, B).

\section{Purified von Willebrand Factor Plus ADAMTS-13 During Shear Stress Conditions}

Prior to shear stress, purified vWF protein plus recombinant ADAMTS-13 protein again demonstrated intact vWF multimers of all sizes and a relative absence of
vWF degradation fragments. However, in the presence of ADAMTS-13, exposure to supraphysiologic shear stress completely eliminated high-molecular-weight vWF multimers and greatly increased vWF degradation fragments (Figure 2, $A$ and $C$ ). The same $11 \mathrm{vWF}$ degradation fragment bands that were observed in patients with an LVAD were significantly $(P<.05)$ elevated. This is in contrast to the changes seen when ADAMTS-13 was absent (Figure 2, $A$ and $B$ ).

\section{ADAMTS-13 Activity During Shear Stress Conditions}

After exposure to shear stress, the activity of recombinant ADAMTS-13 increased from 606 to $1349 \mathrm{ng} / \mathrm{mL}$ (Figure 2, D).

\section{DISCUSSION}

Our findings demonstrate clearly for the first time that there is both a mechanical and a separate enzymatic 


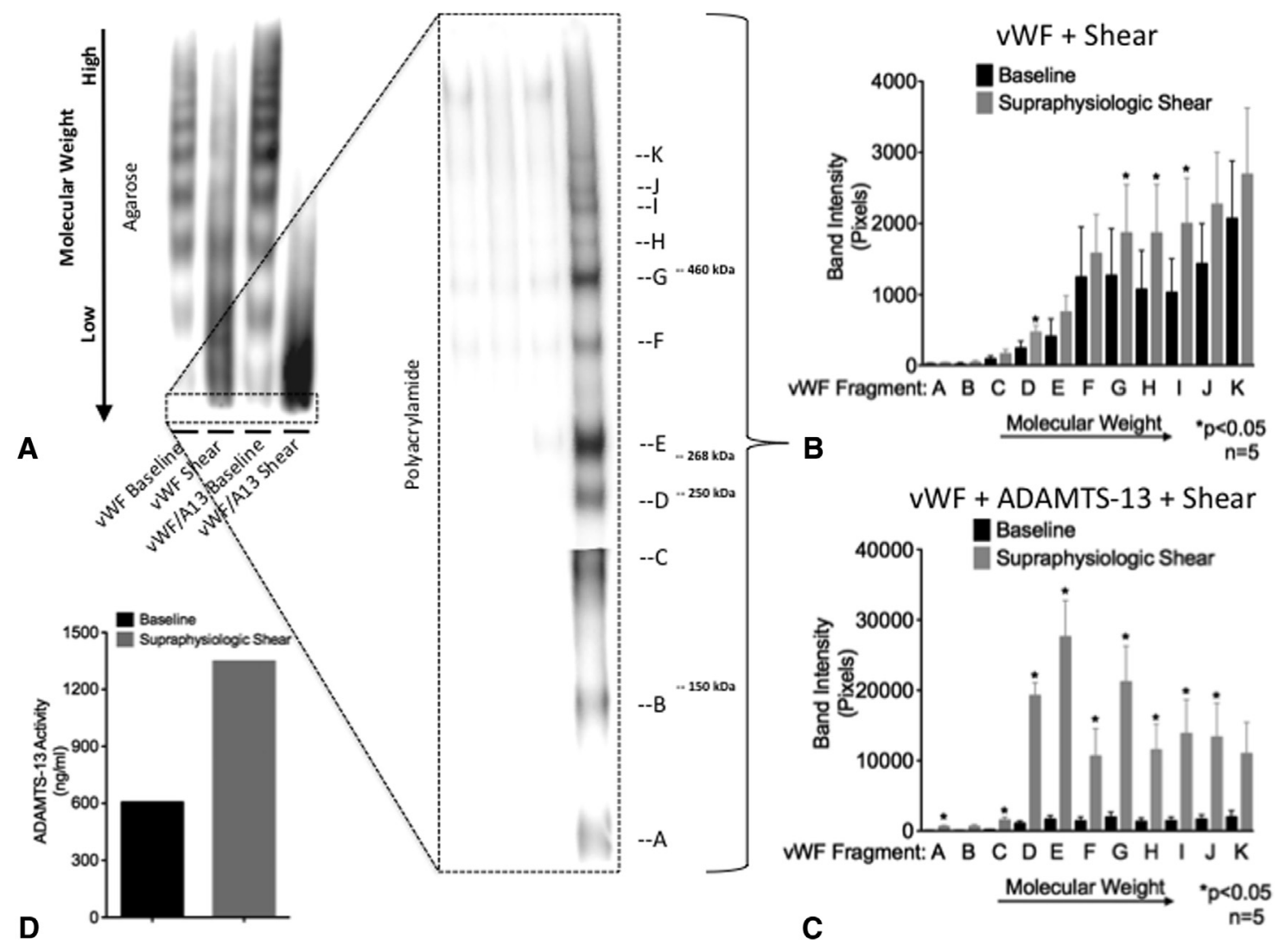

FIGURE 2. A, To investigate the specific mechanistic roles of shear stress and ADAMTS-13 (the vWF protease), purified vWF protein \pm recombinant ADAMTS-13 protein were exposed to supraphysiologic shear stress. vWF multimers and degradation fragments were characterized with electrophoresis and immunoblotting. Exposure of purified vWF alone to supraphysiologic shear stress reduced large vWF multimers and increased small vWF multimers. However, vWF degradation fragments were not generated. In contrast, in the presence of ADAMTS-13, exposure to supraphysiologic shear stress completely eliminated large vWF multimers and significantly increased vWF degradation fragments. B and C, The pattern and quantity of vWF degradation fragments were different when vWF protein was exposed to supraphysiologic shear stress alone versus in the presence of ADAMTS-13 protease. D, In the presence of shear stress, the activity of ADAMTS-13 protein increased. ADAMTS-13, A disintegrin and metalloproteinase with a thrombospondin type 1 motif, member 13; $v W F$, von Willebrand factor; $k D a$, kilodalton.

mechanism of vWF degradation during mechanical circulatory support. This study (1) characterized the profile of vWF multimers and vWF degradation fragments that are generated in vivo in patients with an LVAD; (2) developed and validated an in vitro model that produced the same profile of vWF degradation fragments as a continuous-flow LVAD; (3) demonstrated that supraphysiologic shear stress alone caused a modest mechanical demolition of vWF multimers but did not generate degradation fragments; (4) demonstrated that under conditions of supraphysiologic shear stress, purified vWF plus recombinant ADAMTS-13 (the vWF protease) produced the same profile of vWF degradation fragments as a patient with an LVAD; and (5) demonstrated that the activity of ADAMTS-13 increased supraphysiologic shear stress. These findings highlight the mechanistic importance of both shear stress and ADAMTS-13 in the pathogenesis of acquired vWF deficiency during LVAD therapy (Figure 3). In the setting of shear stress, ADAMTS-13 is the major mechanism of
vWF degradation during mechanical circulatory support. ADAMTS-13 may be an appropriate clinical target to reduce vWF degradation and bleeding in patients with an LVAD. Of great interest, ADAMTS-13 may be inhibited by a number of pharmacologic and biologic agents and may be a potential target to reduce pathologic degradation of vWF during LVAD support.

\section{Normal Mechanisms of von Willebrand Factor Degradation: Shear Stress and ADAMTS-13}

vWF, a 500 to $20,000 \mathrm{kDa}$ multimeric glycoprotein assembled from $225 \mathrm{kDa}$ monomers, is constitutively manufactured by endothelial cells, circulates in the plasma, and participates in primary hemostasis. ${ }^{20} \mathrm{vWF}$ metabolism is closely regulated by the rheologic conditions of the blood. Specifically, shear stress is an important biomechanical trigger of vWF metabolism. At sites of endothelial damage, turbulence and altered shear stress induce a conformational unraveling of vWF that facilitates $\mathrm{vWF}$ tethering to 


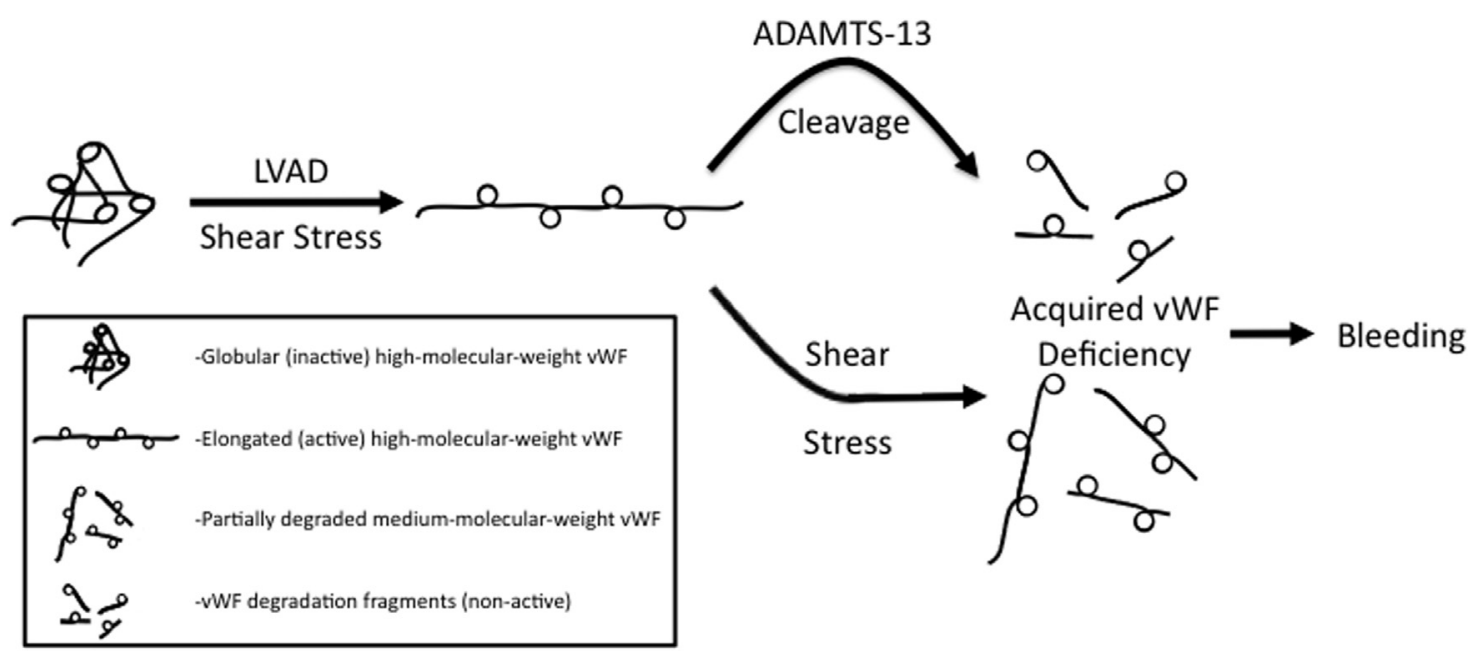

FIGURE 3. Supraphysiologic shear stress during LVAD support triggers globular (inactive) vWF to undergo a conformational elongation into an active form. Supraphysiologic shear stress alone physically demolishes large, active vWF multimers into smaller vWF multimers. In parallel, the major mechanism of vWF degradation with an LVAD is ADAMTS-13 cleavage of large, active vWF multimers into small, nonactive vWF fragments. As a result, an acquired vWF deficiency predisposes patients with an LVAD to bleeding events. ADAMTS-13, A disintegrin and metalloproteinase with a thrombospondin type 1 motif, member 13; LVAD, left ventricular assist device; $v W F$, von Willebrand factor.

subendothelial collagen and platelets. ${ }^{21}$ As a result, a platelet plug covers the breached endothelium. During this process, large vWF multimers are most hemostatically active and most responsive to activation by shear stress. ${ }^{22}$ However, shear-induced activation also renders vWF more sensitive to proteolytic cleavage by ADAMTS-13, ${ }^{23}$ the vWF-specific plasma metalloprotease. As such, abnormal shear stress is a powerful trigger for ADAMTS-13 cleavage of vWF.

A clinical relationship between abnormal shear stress and degradation of vWF is well described. ${ }^{20}$ In patients with critical aortic stenosis, increased shear stress through the stenotic aortic annulus reduces large vWF multimers. ${ }^{24}$ Within hours of aortic valve replacement (and reestablishment of normal shear stress), large vWF multimers return. These findings highlight in humans the clinical importance of physiologic processes that alter shear stress and thereby vWF metabolism.

\section{Clinical Findings}

LVADs significantly alter shear stress. The normal range of shear stress in the human body is 15 to $40 \mathrm{dyne} / \mathrm{cm}^{2}{ }^{17}$ Continuous-flow LVADs are reported to generate shear stress 1 to 2 orders of magnitude greater than physiologic values. ${ }^{16}$ As a result, the blood of a patient with an LVAD is continually exposed to supraphysiologic shear stress. Strong clinical evidence demonstrates that LVADs alter vWF metabolism and predispose patients to bleeding events. $^{2,25}$ Consequently, it has been proposed that shear stress from the LVAD leads to pathologic degradation of vWF and an acquired von Willebrand syndrome. ${ }^{26}$ Animal data support this hypothesis. ${ }^{11,12}$
Our current data demonstrated that patients with an LVAD developed a consistent profile of reduced highmolecular-weight vWF multimers and significantly increased $\mathrm{vWF}$ degradation fragments. It is likely that supraphysiologic shear stress from the LVAD triggered abnormal vWF metabolism. To elucidate mechanism, we performed a series of experiments to isolate the effects of shear stress alone and ADAMTS-13 in the presence of shear stress on vWF degradation. These experiments were performed independently of the effects of blood cells, clotting factors, enzymes, or plasma proteins.

\section{Physical Demolition of von Willebrand Factor by Shear Stress During Left Ventricular Assist Device Support}

To investigate the specific mechanistic roles of shear stress and ADAMTS-13, we developed a tightly controlled in vitro model to reproduce the pathologic vWF degradation of an LVAD. When normal human plasma was exposed to supraphysiologic shear stress in the vortexer, the profile of vWF multimers and degradation fragments was nearly identical to that of a patient with an LVAD (Figure 1), with one important difference. The percent change for band D was significantly greater in the vortexer samples versus the patients with an LVAD. The D band represents the $225 \mathrm{kDa}$, single vWF monomer, a vWF fragment that is not generated by ADAMTS-13 cleavage. This finding suggested that shear stress is a separate mechanism from ADAMTS-13 cleavage for degrading large vWF multimers into smaller fragments with breakpoints at the monomer linkages. In contrast, ADAMTS-13 cleavage generates a $176 \mathrm{kDa}$ and a $140 \mathrm{kDa}$ fragment. 
To define the specific mechanistic role of shear stress alone, purified vWF protein was exposed to supraphysiologic shear stress (independent of ADAMTS-13) (Figure 2, $A$ and $B$ ). Degradation of high-molecularweight vWF multimers into low-molecular weight multimers was observed. However, vWF fragments were not generated in large quantities. These findings suggested that shear stress itself (independent of ADAMTS-13) caused large multimers to break into smaller multimers but not the smallest vWF degradation fragments. As such, shear stress alone is a minor (but still significant) mechanism of vWF degradation during LVAD support.

The physical mechanism by which vWF multimers were dismantled into smaller multimers is unknown. Highmolecular-weight vWF multimers contain numerous disulfide bridges, which are $40 \%$ weaker than carbon-carbon covalent bonds. As a result, a mechanical weak point exists between each vWF monomer in a vWF multimer. We speculate that supraphysiologic shear stress from LVADs may tear apart vWF monomers at the sites of disulfide bonds. This hypothesis was not specifically tested but is conceptually appealing. If this proves to be the case, it will be important to determine the shear stress threshold with an LVAD after which mechanical demolition of vWF becomes biologically apparent and clinically relevant.

\section{Enzymatic Degradation of von Willebrand Factor by ADAMTS-13 During Left Ventricular Assist Device Support}

Previously, the role of ADAMTS-13 in LVAD-induced acquired von Willebrand syndrome was unclear. Limited data were conflicting. Clinical observations suggested that ADAMTS-13 did not play a major role in vWF degradation and bleeding during mechanical circulatory support. ${ }^{5,6,10}$ However, these studies were conducted in small subgroups of patients, lacked appropriate controls, and measured plasma ADAMTS-13 levels, not activity of ADAMTS-13 or ADAMTS-13 cleavage products (vWF fragments). In contrast, preliminary experimental evidence from bovine studies has supported the hypothesis that ADAMTS-13 participates in vWF degradation during LVAD therapy. ${ }^{11,12}$

Our current findings demonstrated that vWF fragments generated in vivo during LVAD support were identical to the vWF fragments that developed when vWF multimers were exposed to supraphysiologic shear stress in the presence of ADAMTS-13 (Figure 2, $A$ and $C$ ). Shear stress increased the enzymatic activity of ADAMTS-13. These findings strongly supported the hypothesis that shear stress from the LVAD plus proteolytic cleavage of vWF by ADAMTS-13 both played a significant role in $\mathrm{vWF}$ degradation during LVAD support. However, in the setting of shear stress, ADAMTS-13 was the major mechanism of vWF degradation.

The vWF fragment bands that demonstrated the greatest increase appeared to be approximately $140 \mathrm{kDa}$ (band B),
$176 \mathrm{kDa}$ (band C), $225 \mathrm{kDa}$ (band D), $300 \mathrm{kDa}$ (band E), $400 \mathrm{kDa}$ (band F), $450 \mathrm{kDa}$ (band G), greater than 460 $\mathrm{kDa}$ (band $\mathrm{H}$ ), and greater than $460 \mathrm{kDa}$ (band $\mathrm{J}$ ) (Figure 1, A). The $225 \mathrm{kDa}$ band likely represented individual vWF monomer subunits, ${ }^{20}$ which also have been observed to be significantly generated in vitro in a mock circulatory loop with an LVAD. ${ }^{11}$ Likewise, the $450 \mathrm{kDa}$ band likely represented the smallest vWF multimer configuration of $2225 \mathrm{kDa}$ monomers joined together.

It is well established that ADAMTS-13 proteolysis of a single $\mathrm{vWF}$ monomer or the terminal monomer on a vWF multimer generates 140 and $176 \mathrm{kDa}$ fragments. ${ }^{27,28}$ As expected, we observed a significant increase in the 140 and $176 \mathrm{kDa} v W F$ fragment bands. Of greater interest, we observed multiple bands that were $400+\mathrm{kDa}$. These bands likely represent different chain combinations of vWF monomers plus a 140 or $176 \mathrm{kDa}$ (or both) fragment attached to the peptide terminal end(s). This finding was consistent among patients with an LVAD and in the in vitro experiments with human blood and purified vWF and ADAMTS-13 protein. Again, this strongly suggested that in the setting of shear stress, ADAMTS-13 was the major mechanism of vWF degradation in patients with an LVAD.

\section{Clinical Implications}

Multiple LVADs are clinically available or poised for clinical trials. ${ }^{29}$ Each device produces a unique hemodynamic profile of abnormal shear stress, pulsatility, and turbulence. ${ }^{30}$ Of note, each device also produces a unique footprint of (patho)physiologic changes to hemostatic pathways. ${ }^{31}$ As such, next-generation LVADs designed to minimize shear stress and mimic native cardiovascular hemodynamics may reduce vWF degradation and bleeding in patients with an LVAD. This may be an unrealistic immediate solution because of economic and time constraints, and other therapies should be investigated.

Our findings suggest that ADAMTS-13 may be a suitable clinical target to reduce vWF degradation during LVAD support. ADAMTS-13 inhibition may serve a therapeutic role in the clinical management of bleeding in patients with an LVAD. Of note, tetracycline antibiotics inhibit matrix metalloproteinases, ${ }^{32}$ which include the ADAMTS protease family. Specifically, doxycycline inhibits ADAMTS $-13^{33}$ and may be worth investigating as a pharmacotherapy to reduce vWF degradation in patients with an LVAD. Prolonged doxycycline therapy is safe and well tolerated by patients for the long-term (months to years) management of inflammatory acnes. ${ }^{34}$ Therefore, it is conceptually appealing to consider that prophylactic administration of doxycycline in patients with an LVAD would be safe and may reduce pathologic degradation of vWF. As an additional benefit, doxycycline may protect patients with an LVAD against aerobic skin flora, such 
as methicillin-resistant Staphylococcus aureus, which frequently causes LVAD-related infections. Further experimental studies are warranted to investigate ADAMTS-13 inhibition with doxycycline, ${ }^{33}$ interleukin- $6,{ }^{35}$ and plasma free hemoglobin ${ }^{36}$ as a novel pathway to reduce LVADassociated vWF degradation.

\section{CONCLUSIONS}

Our findings demonstrate the mechanistic importance of shear stress and ADAMTS-13 (the vWF protease) in the pathologic degradation of vWF during mechanical circulatory support. Supraphysiologic shear stress itself caused moderate degradation of vWF multimers but did not generate vWF fragments. In the presence of supraphysiologic shear stress, ADAMTS-13 (the vWF protease) eliminated high-molecular-weight vWF multimers and generated vWF degradation fragments. As such, ADAMTS-13 is the major mechanism of vWF degradation during mechanical circulatory support. However, both mechanistic pathways likely contribute to bleeding episodes in patients with an LVAD. ADAMTS-13 may be a therapeutic target to reduce vWF degradation and predisposition to bleeding in patients with an LVAD. ADAMTS-13 inhibitors should be investigated as a potential intervention to reduce pathologic degradation of vWF during mechanical circulatory support.

The authors thank Dr Joseph Woo, Jooeun Kang, George Hung, Alen Trubelja, and the mechanical circulatory support team at the University of Pennsylvania. Jessica Howard was instrumental in obtaining institutional review board approval for this project. Dr Robert Dowling and Sujith Dassanayaka provided valuable feedback during drafting of the manuscript.

\section{References}

1. Kirklin JK, Naftel DC, Kormos RL, Stevenson LW, Pagani FD, Miller MA, et al. Fifth INTERMACS annual report: risk factor analysis from more than 6,000 mechanical circulatory support patients. J Heart Lung Transplant. 2013;32:141-56.

2. Islam S, Cevik C, Madonna R, Frandah W, Islam E, Islam S, Nugent K. Left ventricular assist devices and gastrointestinal bleeding: a narrative review of case reports and case series. Clin Cardiol. 2013;36:190-200.

3. Heilmann C, Geisen U, Beyersdorf F, Nakamura L, Trummer G, BerchtoldHerz M, et al. Acquired Von Willebrand syndrome is an early-onset problem in ventricular assist device patients. Eur J Cardiothorac Surg. 2011;40:1328-33.

4. Uriel N, Pak SW, Jorde UP, Jude B, Susen S, Vincentelli A, et al. Acquired von Willebrand syndrome after continuous-flow mechanical device support contributes to a high prevalence of bleeding during long-term support and at the time of transplantation. J Am Coll Cardiol. 2010;56:1207-13.

5. Klovaite J, Gustafsson F, Mortensen SA, Sander K, Nielsen LB. Severely impaired von Willebrand factor-dependent platelet aggregation in patients with a continuous-flow left ventricular assist device (HeartMate II). J Am Coll Cardiol. 2009;53:2162-7.

6. Crow S, Milano C, Joyce L, Chen D, Arepally G, Bowles D, et al. Comparative analysis of von Willebrand factor profiles in pulsatile and continuous left ventricular assist device recipients. ASAIO J. 2010;56:441-5.

7. Geisen U, Heilmann C, Beyersdorf F, Benk C, Berchtold-Herz M, Schlensak C, et al. Non-surgical bleeding in patients with ventricular assist devices could be explained by acquired von Willebrand disease. Eur J Cardiothorac Surg. 2008;33:679-84.
8. Steinlechner B, Dworschak M, Birkenberg B, Duris M, Zeidler P, Fischer H, et al. Platelet dysfunction in outpatients with left ventricular assist devices. Ann Thorac Surg. 2009;87:131-7.

9. Crow S, Chen D, Milano C, Thomas W, Joyce L, Piacentino V III, et al. Acquired von Willebrand syndrome in continuous-flow ventricular assist device recipients. Ann Thorac Surg. 2010;90:1263-9.

10. Meyer AL, Malehsa D, Bara C, Budde U, Slaughter MS, Haverich A, Strueber M. Acquired von Willebrand syndrome in patients with an axial flow left ventricular assist device. Circ Heart Fail. 2010;3:675-81.

11. Dassanayaka S, Slaughter MS, Bartoli CR. Mechanistic pathway(s) of acquired von Willebrand syndrome with a continuous-flow ventricular assist device: in vitro findings. ASAIO J. 2013;59:123-9.

12. Bartoli CR, Dassanayaka S, Brittian KR, Luckett A, Sithu S, Siess T, et al. Insights into the mechanism(s) of von Willebrand factor degradation during mechanical circulatory support. J Thorac Cardiovasc Surg. 2014;147:1634-43.

13. Restle DJ, Zhang DM, Hung G, Howard JL, Kallel F, Acker MA, Atluri P, Bartoli CR. Preclinical models for translational investigations of left ventricular assist device-associated von Willebrand factor degradation. Artif Organs. 2014 [in press].

14. Salek MM, Sattari P, Martinuzzi RJ. Analysis of fluid flow and wall shear stress patterns inside partially filled agitated culture well plates. Ann Biomed Eng. 2012; 40:707-28.

15. Dardik A, Chen L, Frattini J, Asada H, Aziz F, Kudo FA, Sumpio BE. Differential effects of orbital and laminar shear stress on endothelial cells. J Vasc Surg. 2005; 41:869-80.

16. Fraser KH, Zhang T, Taskin ME, Griffith BP, Wu ZJ. A quantitative comparison of mechanical blood damage parameters in rotary ventricular assist devices: shear stress, exposure time and hemolysis index. J Biomech Eng. 2012;134:081002.

17. Resnick N, Yahav H, Shay-Salit A, Shushy M, Schubert S, Zilberman LC, Wofovitz E. Fluid shear stress and the vascular endothelium: for better and for worse. Prog Biophys Mol Biol. 2003;81:177-99.

18. Starke RD, Ferraro F, Paschalaki KE, Dryden NH, McKinnon TA, Sutton RE, et al. Endothelial von Willebrand factor regulates angiogenesis. Blood. 2011; 117:1071-80.

19. Diagnostica A. ACTIFLUOR ADAMTS-13 Activity Assay. American Diagnostica, Stamford, CT; Ref 812.

20. Sadler JE. Biochemistry and genetics of von Willebrand factor. Annu Rev Biochem. 1998;67:395-424.

21. Siedlecki CA, Lestini BJ, Kottke-Marchant KK, Eppell SJ, Wilson DL, Marchant RE. Shear-dependent changes in the three-dimensional structure of human von Willebrand factor. Blood. 1996;88:2939-50.

22. Zhang X, Halvorsen K, Zhang CZ, Wong WP, Springer TA. Mechanoenzymatic cleavage of the ultralarge vascular protein von Willebrand factor. Science. 2009; 324:1330-4.

23. Tsai HM, Sussman II, Nagel RL. Shear stress enhances the proteolysis of von Willebrand factor in normal plasma. Blood. 1994;83:2171-9.

24. Vincentelli A, Susen S, Le Tourneau T, Six I, Fabre O, Juthier F, et al. Acquired von Willebrand syndrome in aortic stenosis. N Engl J Med. 2003;349:343-9.

25. Crow S, John R, Boyle A, Shumway S, Liao K, Colvin-Adams M, et al. Gastrointestinal bleeding rates in recipients of nonpulsatile and pulsatile left ventricular assist devices. J Thorac Cardiovasc Surg. 2009;137:208-15.

26. Tsai HM. von Willebrand factor, shear stress, and ADAMTS13 in hemostasis and thrombosis. ASAIO J. 2012;58:163-9.

27. Dent JA, Galbusera M, Ruggeri ZM. Heterogeneity of plasma von Willebrand factor multimers resulting from proteolysis of the constituent subunit. J Clin Invest. 1991;88:774-82.

28. Turner NA, Nolasco L, Ruggeri ZM, Moake JL. Endothelial cell ADAMTS-13 and VWF: production, release, and VWF string cleavage. Blood. 2009;114:5102-11.

29. Bartoli CR, Dowling RD. The future of adult cardiac assist devices: novel systems and mechanical circulatory support strategies. Cardiol Clin. 2011;29:559-82.

30. Bartoli CR, Spence PA, Siess T, Raess DH, Koenig SC, Dowling RD. Nonphysiologic blood flow triggers endothelial and arterial remodeling in vivo: implications for novel left ventricular assist devices with a peripheral anastomosis. $J$ Thorac Cardiovasc Surg. 2014; 148:311-21.

31. Birschmann I, Dittrich M, Eller T, Wiegmann B, Reininger AJ, Budde U, Struber M. Ambient hemolysis and activation of coagulation is different between HeartMate II and HeartWare left ventricular assist devices. J Heart Lung Transplant. 2014;33:80-7.

32. Castro MM, Kandasamy AD, Youssef N, Schulz R. Matrix metalloproteinase inhibitor properties of tetracyclines: therapeutic potential in cardiovascular diseases. Pharmacol Res. 2011;64:551-60. 
33. Tsai HM, Sussman II, Ginsburg D, Lankhof H, Sixma JJ, Nagel RL. Proteolytic cleavage of recombinant type $2 \mathrm{~A}$ von Willebrand factor mutants R834W and R834Q: inhibition by doxycycline and by monoclonal antibody VP-1. Blood. 1997;89:1954-62.

34. Kircik LH. Doxycycline and minocycline for the management of acne: a review of efficacy and safety with emphasis on clinical implications. J Drugs Dermatol. 2010;9:1407-11.
35. Bernardo A, Ball C, Nolasco L, Moake JF, Dong JF. Effects of inflammatory cytokines on the release and cleavage of the endothelial cell-derived ultralarge von Willebrand factor multimers under flow. Blood. 2004;104:100-6.

36. Studt JD, Kremer Hovinga JA, Antoine G, Hermann M, Rieger M, Scheiflinger F, Lammle B. Fatal congenital thrombotic thrombocytopenic purpura with apparent ADAMTS13 inhibitor: in vitro inhibition of ADAMTS13 activity by hemoglobin. Blood. 2005;105:542-4.

\section{EDITORIAL COMMENTARY}

\section{A new spin on acquired von Willebrand factor deficiency during continuous-flow left ventricular assist device support}

John M. Toole, MD

See related article on pages 281-9.

In their article in this issue of the Journal of Thoracic and Cardiovascular Surgery, Bartoli and associates ${ }^{1}$ have created a simple in vitro model that mimics left ventricular assist device (LVAD) shear forces to study the mechanisms of breakdown of von Willebrand factor (vWF). The effects of shear force and nonpulsatile blood flow in the setting of severe aortic stenosis have long been recognized to lead to formation of gastrointestinal arteriovenous malformations ${ }^{2}$ and an acquired deficiency of $\mathrm{vWF}^{3}$ In patients supported by continuous-flow LVADs who require aspirin and warfarin therapy to minimize the odds of device thrombosis, this pathophysiologic combination creates a difficult clinical scenario to navigate. Furthermore, a recently observed increase in early pump thrombosis ${ }^{4}$ has heightened concerns regarding balancing the risks of bleeding and thrombosis.

In this study, Bartoli and associates ${ }^{1}$ demonstrated that the pattern of $\mathrm{vWF}$ breakdown into multimers, monomers, and partial fragments seen in the blood of patients with LVADs matches that seen in the blood of healthy volunteers after 4 hours in a vortexer. After validating the vortexer as an in vitro model for an LVAD, they conducted a series of

\footnotetext{
From the Department of Cardiothoracic Surgery, Medical University of South Carolina, Charleston, SC

Disclosures: Author has nothing to disclose with regard to commercial support.

Received for publication Sept 16, 2014; accepted for publication Sept 18, 2014; available ahead of print Oct 14, 2014.

Address for reprints: John M. Toole, MD, Medical University of South Carolina, Cardiothoracic Surgery, 25 Courtenay Dr, ART 7026, Charleston, SC 29425 (E-mail: toole@musc.edu).

J Thorac Cardiovasc Surg 2015;149:289-90

$0022-5223 / \$ 36.00$

Copyright (c) 2015 by The American Association for Thoracic Surgery

http://dx.doi.org/10.1016/j.jtcvs.2014.09.052
}

experiments in which they demonstrated shear force leads to breakdown of large-molecular weight vWF into smaller but functional polymers. In contrast, shear in combination with a disintegrin and metalloproteinase with a thrombospondin type 1 motif, member 13 (ADAMTS-13), the vWF-cleaving metalloprotease, leads to a greater degree of breakdown and fragmentation of monomers, which matches the pattern seen in LVAD-supported patients. These fragments and monomers are not active, leading to vWF deficiency. Furthermore, shear force was observed to increase the activity of ADAMTS-13, thereby amplifying its effect on vWF breakdown. The implication is that pharmacologic antagonism of ADAMTS-13 may restore function of vWF. This is an easily tested hypothesis because of the simplicity of this model.

A well-known antagonist of ADAMTS-13 is doxycycline. Although prevention of infection would be a beneficial side effect of its use, it would be unwise to put this into clinical use before adequate confirmation first in the vortexer model then prospectively in a clinical trial. It is possible that acquired $\mathrm{vWF}$ deficiency is protective with regard to pump thrombosis and that alteration of this hematologic balancing act might be harmful.

The simplicity of this model is what makes it ingenious. Vortexers are ubiquitous in research laboratories worldwide. This model allows relatively quick and inexpensive experimentation that might have otherwise required significant time, laboratory animals, human subjects, or spare or salvaged LVADs. It is to hoped that more valuable information will follow.

\section{References}

1. Bartoli CR, Restle DJ, Zhang DM, Acker MA, Atluri P. Pathological von Willebrand degradation with an LVAD occurs via two distinct mechanisms: mechanical demolition and enzymatic cleavage. J Thorac Cardiovasc Surg. 2015;149:281-9. 2. Heyde EC. Gastrointestinal bleeding in aortic stenosis. N Engl J Med. 1958;259: 196. 